\title{
Opportunistic skeletal muscle metrics as prognostic tools in metastatic castration-resistant prostate cancer patients candidates to receive Radium-223
}

\author{
Matteo Bauckneht ${ }^{1,2}$ - $\cdot$ Rita Lai $^{3}$. Francesca D'Amico ${ }^{1}$ - Alberto Miceli ${ }^{1}$ Maria Isabella Donegani ${ }^{1}$. \\ Cristina Campi ${ }^{4}$. Daniela Schenone ${ }^{4}$ - Stefano Raffa ${ }^{1}$ - Silvia Chiola ${ }^{2}$. Francesco Lanfranchi ${ }^{1}$ - Sara Elena Rebuzzi ${ }^{5,6}$. \\ Elisa Zanardi ${ }^{7} \cdot$ Malvina Cremante $^{8} \cdot$ Cecilia Marini $^{2,9} \cdot$ Giuseppe Fornarini $^{8} \cdot$ Silvia Morbelli $^{1,2} \cdot$ Michele Piana $^{4,10}$. \\ Gianmario Sambuceti ${ }^{1,2}$
}

Received: 27 December 2021 / Accepted: 7 January 2022 / Published online: 19 January 2022

(c) The Author(s) 2022

\begin{abstract}
Objective Androgen deprivation therapy alters body composition promoting a significant loss in skeletal muscle (SM) mass through inflammation and oxidative damage. We verified whether SM anthropometric composition and metabolism are associated with unfavourable overall survival (OS) in a retrospective cohort of metastatic castration-resistant prostate cancer (mCRPC) patients submitted to 18F-Fluorodeoxyglucose Positron Emission Tomography/Computed Tomography (FDG PET/CT) imaging before receiving Radium-223.

Patients and methods Low-dose CT were opportunistically analysed using a cross-sectional approach to calculate SM and adipose tissue areas at the third lumbar vertebra level. Moreover, a 3D computational method was used to extract psoas muscles to evaluate their volume, Hounsfield Units (HU) and FDG retention estimated by the standardized uptake value (SUV). Baseline established clinical, lab and imaging prognosticators were also recorded.

Results SM area predicted OS at univariate analysis. However, this capability was not additive to the power of mean HU and maximum SUV of psoas muscles volume. These factors were thus combined in the Attenuation Metabolic Index (AMI) whose power was tested in a novel uni- and multivariable model. While Prostate-Specific Antigen (PSA), Alkaline Phosphatase (ALP), Lactate Dehydrogenase and Hemoglobin, Metabolic Tumor Volume, Total Lesion Glycolysis and AMI were associated with long-term OS at the univariate analyses, only PSA, ALP and AMI resulted in independent prognosticator at the multivariate analysis.

Conclusion The present data suggest that assessing individual 'patients' SM metrics through an opportunistic operatorindependent computational analysis of FDG PET/CT imaging provides prognostic insights in mCRPC patients candidates to receive Radium-223.
\end{abstract}

Matteo Bauckneht

matteo.bauckneht@gmail.com

1 Department of Health Sciences (DISSAL), University of Genova, Genova, Italy

2 Nuclear Medicine, IRCCS Ospedale Policlinico San Martino, Genova, Italy

3 Department of Mathematics (DIMA), University of Genoa, Genoa, Italy

4 LISCOMP, Department of Mathematics (DIMA), University of Genoa, Genoa, Italy

5 Medical Oncology, Ospedale San Paolo, Savona, Italy
6 Department of Internal Medicine and Medical Specialties (Di.M.I.), University of Genova, Genoa, Italy

7 Academic Unit of Medical Oncology, IRCCS Ospedale Policlinico San Martino, Genoa, Italy

8 Medical Oncology Unit 1, IRCCS Ospedale Policlinico San Martino, Genoa, Italy

9 Bioimaging and Physiology (IBFM), CNR Institute of Molecular, Segrate, Milan, Italy

10 CNR-SPIN Genoa, Genoa, Italy 


\section{Graphical abstract}

Study design and methods

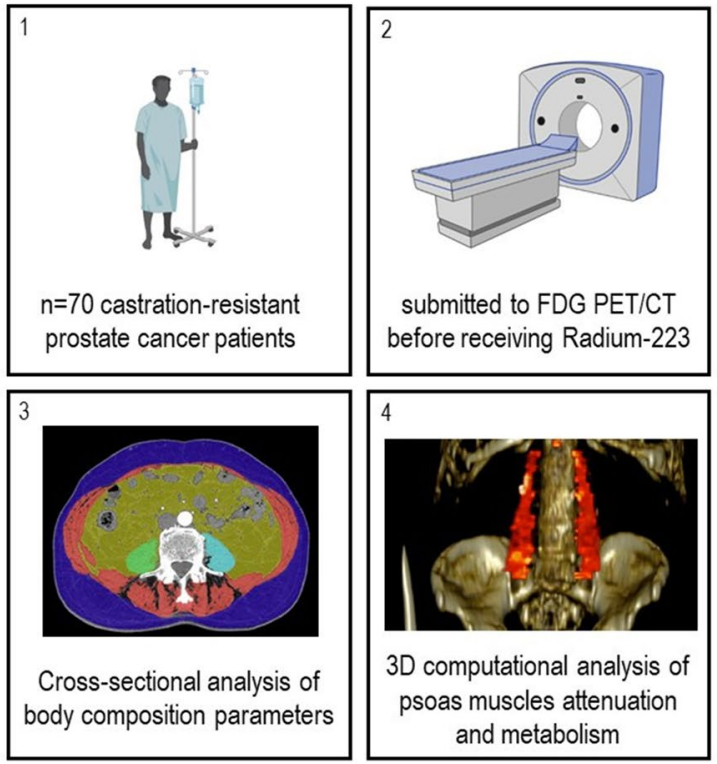

Study results

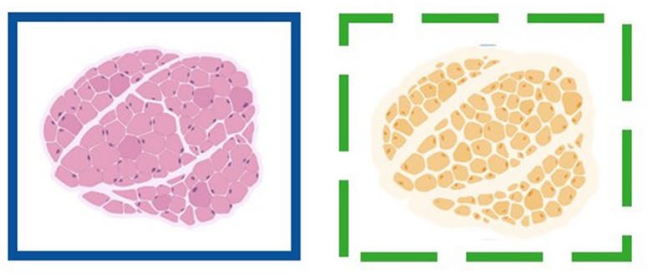

High density, low FDG uptake Low density, high FDG uptake

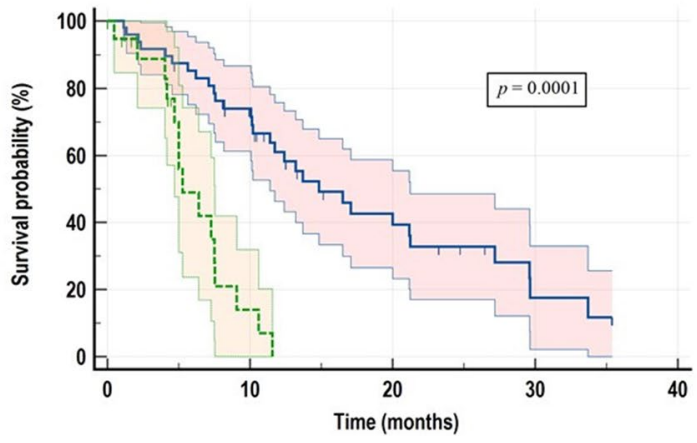

Keywords Metastatic castration-resistant prostate cancer $\cdot$ Skeletal muscle $\cdot$ Sarcopenia $\cdot$ 18F-Fluorodeoxyglucose $\cdot$ Positron emission tomography $\cdot$ Radium-223

\section{Introduction}

Prostate cancer (PC) represents the most common solid male malignancy in the western world [1]. The clinical behaviour of PC is widely heterogeneous, ranging from hormone-responsive disease to a highly aggressive and androgen-deprivation therapy (ADT), including the administration of alpha-emitting radionuclides such as Radium-223 [3-9].

The phase III Alpharadin in Symptomatic Prostate Cancer Patients (ALSYMPCA) trial recruited patients with mCRPC with exclusive bone metastases who received Radium-223 compared to placebo, showing a significantly improved OS in the treated group [10]. Nevertheless, this result was only partially confirmed in clinical practice during the last decade [11-13]. Among the possible explanations of this discrepancy, concerns have been raised about the criteria used for the selection of candidates for this treatment [14]. Therefore, defining prognostic factors able to identify mCRPC patients who will most likely benefit from Radium-223 since baseline, potentially improving this difficult selection process, represents a challenging and crucial issue.

In this scenario, a few studies showed that imaging of ${ }^{18} \mathrm{~F}$ Fluorodeoxyglucose (FDG) uptake with Positron Emission Tomography/Computed Tomography (PET/CT) provides prognostic insights in advanced $\mathrm{mCRPC}$, potentially guiding treatment-resistant one $[1,2]$. This latter setting is often characterized by a metastatic diffusion combined with the loss of hormone sensitivity configuring the clinical scenario of metastatic castration-resistant prostate cancer (mCRPC). In these patients, several treatment options can improve the overall survival (OS) when added to the the systemic treatment selection [15-17]. In particular, the higher is FDG retention in skeletal lesions, the lower are the chances of response to Radium-223 [16, 17]. Nevertheless, PET/CT imaging may opportunistically provide a series of data related to the normal tissues of MCRPC patients, whose analysis might provide prognostic insights independent of the tumour itself.

Among these variables, sarcopenia recently gained attention as a prognosticator in PC [18-21]. Sarcopenia defines a systematic loss of skeletal muscle (SM) mass that decreases below two standard deviations of normal healthy adults [22]. The analysis of the SM area at the level of the third lumbar vertebra (L3) in CT images has already proved to represent an accurate procedure to identify sarcopenia [23]. However, given the multifactorial aetiology underlying muscle wasting, including systemic inflammation [24], FDG PET/CT might represent a potential better descriptor of this muscle disorder.

On these bases, the present study aimed to assess whether SM mass, SM metabolism or their eventual combination, 
opportunistically derived from FDG PET/CT imaging, are associated with unfavourable OS in a cohort of mCRPC patients' candidates to Radium-223 therapy.

\section{Materials and methods}

\section{Study population and design}

We performed a retrospective analysis of all consecutive mCRPC patients treated with Radium-223 from January 2015 to November 2021 at IRCCS Ospedale Policlinico San Martino of Genoa, Italy. CRPC was defined as a serum testosterone level of $<50 \mathrm{ng} / \mathrm{dl}$ following pharmaceutical castration. All recruited patients were submitted to FDG PET/ CT before receiving Radium-223 for prognostic purposes, as indicated by the national guidelines [25]. The study was performed according to the Declaration of Helsinki, Good Clinical Practice, and local ethical regulations. The local ethical committee of Regione Liguria approved the study (Regional Ethical Committee of Liguria-registration number 590/2020). All patients enrolled in the study signed a written informed consent at the time of FDG PET/CT and at the time of each Radium-223 administration, encompassing the use of anonymized data for retrospective research purposes.

\section{Imaging and treatment procedures}

FDG PET/CT was performed according to the European Association of Nuclear Medicine (EANM) Guidelines [26]. Briefly, after a minimum 6-h fasting, a dose of 4.8-5.2 MBq of FDG per kilogram of body weight was injected through a peripheral vein catheter. Patients were placed in a quiet room and instructed to remain still. Data acquisition started $\geq 60 \mathrm{~min}$ after tracer injection. Patients underwent low-dose CT from the skull base to the thighs for attenuation-correction and anatomic localization of the FDG-avid lesions, followed by PET imaging. PET/CT studies were performed with two different PET/CT systems (Hirez-Biograph 16; Siemens Medical Solutions, Munich, Germany and Biograph mCT Flow; Siemens Medical Solutions, Munich, Germany). Standard parameters used were CT: $80 \mathrm{~mA}, 120 \mathrm{kV}$ without contrast; $2.5 \mathrm{~min}$ per bed-PET-step of $15 \mathrm{~cm}$; the reconstruction was performed in a $128 \times 128$ matrix and with a $60 \mathrm{~cm}$ field-of-view. PET images reconstruction was obtained using ordered subset expectation maximization (OSEM) algorithms, and attenuation correction was performed using the $\mathrm{CT}$ raw data. The entire CT dataset was fused with the 3-dimensional PET images using an integrated software interface (Syngo Image Fusion; Siemens Erlangen, Germany) to create anatomical images superimposed with FDG uptake.
Radium-223 (55 KBq/kg) was intravenously administered every four weeks and continued until disease progression, unacceptable toxicity, death, or patient choice up to six cycles. According to the current EANM guidelines, chemotherapy, Abiraterone, or Enzalutamide were discontinued before the first Radium-223 administration, while patients continued receiving androgen deprivation therapy [27]. As part of our standard protocol, patients were clinically followed-up until death or patient choice after treatment completion.

\section{Anthropometric measurements on FDG PET/CT images}

Anthropometric measurements were performed on PET/ CT images using a cross-sectional and a 3D computational approach.

The cross-sectional areas of subcutaneous fat (HU: -190 to -30 ), visceral fat (HU: -150 to -50 ), and SM (HU: -29 to 150) from the low-dose CT of PET/CT scan at the level of L3 were calculated using a validated freely available online software (www.CoreSlicer.com) [28]. For SM cross-sectional analysis, two consecutive images were recorded in the L3 plane. The sum of the cross-sectional areas of all SM was calculated, averaged, and divided by the square of the height (termed Skeletal Muscle Index, SMI). The formula used was: SMI $=$ L3 SM cross-sectional area $\left(\mathrm{cm}^{2}\right) /$ height $^{2}\left(\mathrm{~m}^{2}\right)$ [29]. The occurrence of sarcopenia was defined according to international consensus definitions of an SMI $<55 \mathrm{~cm}^{2} /$ $\mathrm{m}^{2}$ for men [29]. Adipose tissue area was calculated as the sum between visceral and subcutaneous fat areas. Visceralto-subcutaneous fat ratio (VSR) and visceral fat-to-muscle ratio (VMR) were also calculated.

To improve the accuracy of the anthropometric evaluation, an in-house validated operator-independent 3D computational approach based on the Hough transform was also applied [30, 31]. This method can extract the entire recognizable psoas muscles volume from the low-dose CT images of PET/CT scans instead of a single muscle slice at the level of L3. This approach extracts the Psoas volume from its insertion in L2-L3 to the L5-S1 symphysis plane. Extracted volumes, average Psoas HU (HUmean) and HUmean Standard Deviation (HUmean SD) can be thus extracted from the entire muscles in an operator-independent fashion. The extracted binary mask can also be translated to the corresponding FDG PET data to calculate maximum and mean Standardized Uptake Values (SUV) from the entire Psoas muscles volumes of interest.

\section{Prognostic clinical and imaging data collection}

Baseline established prognosticator included patient's age, Gleason Score (GS), International Society of Urological Pathology (ISUP) grade group at diagnosis, serum 
Prostate-Specific Antigen (PSA), alkaline phosphatase (ALP) and lactate dehydrogenase (LDH) at diagnosis and at the time of imaging, the number of bone metastases at bone scan, the number of previous lines of systemic treatment, and the eventual prior chemotherapy. The maximum standardized uptake value (SUVmax) of the hottest metastatic lesion was obtained from FDG PET/CT images. A volume of interest was then drawn using an SUV-based automated contouring program with an isocounter threshold based on $40 \%$ of the SUVmax [32]. The sum of all metastatic lesions identified the total Metabolic Tumor Volume (MTV). In contrast, the sum of the products between volume and the corresponding SUVmean of each lesion determined the Total Lesion Glycolysis (TLG).

\section{Statistical analyses}

The descriptive analyses were conducted using absolute frequency and percentage for categorical variables and by median and range for quantitative variables. Continuous data are expressed as mean \pm SD. The study's primary endpoint was the overall survival (OS), which was defined as the time from FDG PET/CT imaging until death from any cause, censored at last follow-up for patients who were alive. The Kaplan-Meier (KM) method was used to estimate the survival curve of OS [33]. Differences were considered statistically significant when the $p$ value $(p)$ was $<0.05$. Univariate and multivariate analyses were performed, assessing clinical, laboratory and imaging parameters in correlation with OS, using Cox proportional hazard regression model, estimating hazard ratios (HRs) and their 95\% confidence interval (CI). Only factors with a $p<0.10$ at the univariable analysis were evaluated in the multivariable analyses for OS. All statistical analyses were performed using the software IBM-SPSS release 23 (IBM, Armonk, USA) and MedCalc release 12 (MedCalc Software, Mariakerke, Belgium).

\section{Results}

\section{Patients' characteristics}

Seventy mCRPC patients were included in the analysis. Patients' and treatment characteristics are summarised in Table 1 . The mean age was $74.81 \pm 8.4$ years, and patients with $\geq 75$ years were $45.7 \%$ of the entire cohort. At the time of diagnosis, 35/70 (50\%) and 37/70 (53\%) had GS $\geq 8$ and were metastatic, respectively. Among all patients, 19\% received Radium-223 as second-line therapy, while 33.3\% and $47.6 \%$ received Radium-223 as the third or further line, respectively. Most patients had previously received chemotherapy (58.7\%). At the time of data cut-off (November 2021), with a median follow-up of 8.6 months, $46 / 70$
(65.7\%) of patients were dead, and the median OS (mOS) was 11.5 months (Fig. 1).

\section{Cross-sectional versus computational analysis of SM composition}

All 70 mCRPC enrolled patients were classified as radiologically sarcopenic and the mean SMI of the study cohort was $34.27 \pm 6.2$. Results from univariate and multivariate analyses, including body weight, BMI, cross-sectional and 3D computational body composition parameters, are reported in Table 2. Bodyweight and BMI did not correlate with OS. Among the cross-sectional body composition parameters, SM area significantly correlated with OS, while SMI, adipose tissue area, VSR, and VMR did not reach significance. Among the 3D computational body composition parameters, psoas HUmean and psoas SUVmax resulted prognostic, while psoas volume, psoas HUmean SD and Psoas SUVmean did not predict OS.

Specifically, lower SM area, low HUmean and high SUVmax were associated with a poor OS, suggesting that the inferior long-term outcome is predicted by the reduction in SM body content, SM density as well as by the increased SM FDG uptake. The multivariate model including SM area $\left(<102.8\right.$ vs $\left.\geq 102.8 \mathrm{~cm}^{2}\right)$, psoas HUmean $(<29.5$ vs $\geq 29.5$ ), and psoas SUVmax ( $<3.9$ vs $\geq 3.9$ ) identified psoas HUmean and SUVmax as independent predictors of longterm OS (both with $\mathrm{p} \leq 0.01$ ).

\section{Association of structural and metabolic SM composition parameters and $0 S$}

Results from Kaplan-Meier analyses of HUmean and SUVmax, are reported in Fig. 2A, B. The combination of the parameters mentioned above allowed us to identify a novel composite index, which categorized the enrolled cases in two groups with different risks as it follows: low risk (including cases with neither HUmean $<29.55$ nor SUVmax $>3.89$ and cases with HUmean $<29.55$ or SUV$\max >3.89, n=49$ ), and high risk (HUmean $<29.55$ and SUVmax $>3.89, n=21$ ). Given both attenuation and metabolic parameters as determinants, the obtained index was named Attenuation Metabolic Index (AMI). Kaplan-Meier curves for AMI are represented in Fig. 2C. Median OS was 16.5 months (95\% CI 9.03-23.97 months), and 5.6 months (95\% CI 3.81-7.45 months) for the low and the high AMI groups, respectively $(p<0.0001)$. When included in a multivariate model containing acknowledged clinical, lab and imaging prognosticators, AMI resulted in an independent predictor of long-term OS (Table 3). 
Table 1 Patients' characteristics

\begin{tabular}{ll}
\hline Clinical characteristics & $n(\%)$ \\
\hline Median age, years (range) & $74.77(51.7-89.1)$ \\
Median weight, kg (range) & $80.00(54-119)$ \\
Median Body Mass Index (BMI) & $26.93(18.5-38.6)$ \\
International Society of Urological Pathology (ISUP) grade group & \\
Gleason Score <8 & $23 / 70(32.8 \%)$ \\
Gleason Score $\geq 8$ & $35 / 70(50 \%)$ \\
Missing & $12 / 70(17.2 \%)$ \\
Metastases at diagnosis & \\
No & $33 / 70(47.2 \%)$ \\
Yes & $37 / 70(52.8 \%)$ \\
Lab tests at diagnosis & \\
Prostate Specific Antigen (PSA, ng/mL) & $329.87 \pm 1561.9$ \\
Prior chemotherapy & \\
No & $12 / 70(17.2 \%)$ \\
Yes & $51 / 70(72.8 \%)$ \\
Missing & $7 / 70(10 \%)$ \\
Lab tests at the time of FDG PET/CT imaging & \\
Hemoglobin (g/dL) & $11.49 \pm 1.8$ \\
Prostate Specific Antigen (PSA, ng/mL) & $391.46 \pm 946.7$ \\
Alkaline phosphatase (AP, U/L) & $234.45 \pm 271.72$ \\
Lactate dehydrogenase (LDH, U/L) & $322.36 \pm 322.63$ \\
Neutrophil to lymphocyte ratio (NLR) & $5.24 \pm 4.3$ \\
FDG PET/CT parameters & \\
Standardized Uptake Value (SUVmax) of the hottest bone lesion & $8.19 \pm 4.2$ \\
Metabolic Tumor Volume (MTV, cm ${ }^{3}$ ) & $523.96 \pm 591.4$ \\
Total Lesion Glycolysis (TLG) & $2207.47 \pm 2982.8$ \\
\hline & \\
\hline
\end{tabular}

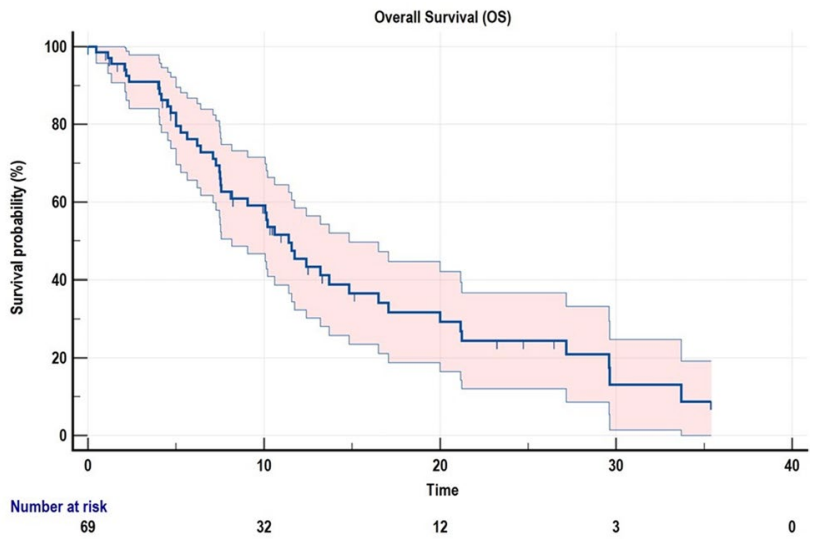

Fig. 1 Kaplan-Meier survival function of the study cohort

\section{Discussion}

A growing body of literature suggests that the efficacy of Radium-223 is closely dependent on pre-treatment 'risk stratification [14]. Therefore, several studies investigated many potential baseline prognostic factors, whose application might optimize the patient's selection process [34]. Coherently with the existing literature, in the present study we observed a prognostic role of baseline PSA, ALP, $\mathrm{LDH}$, and haemoglobin in a cohort of mCRPC candidates to receive Radium-223 [35-41]. Similarly, in agreement with the existing literature [16, 17], the extent and metabolic activity of the metastatic burden, as described by MTV and TLG derived from FDG PET/CT images, predicted OS. On the other hand, we observed that baseline SM structural and metabolic metrics as well as their combination correlate with long-term survival, regardless of the clinical, lab and imaging descriptors of the tumour extension. These data can be opportunistically obtained from FDG PET/CT images, without additional imaging examination costs or radiation exposure.

In the last years, there has been an increasing interest in assessing opportunistic biomarkers from routine, standard of care imaging. An emblematic example is represented by the imaging-based assessment of sarcopenia, which has been correlated with long-term OS in a wide range of solid cancers [42]. The occurrence of sarcopenia has also been related to the increased risk of postsurgical complications [43] and systemic treatment toxicity [44], leading to the notion that 
Table 2 Uni- and multivariable Cox regression analyses including cross-sectional and 3D computational body composition metrics

\begin{tabular}{|c|c|c|c|c|c|}
\hline \multirow[t]{2}{*}{ Variables } & & \multicolumn{2}{|l|}{ Univariate } & \multicolumn{2}{|l|}{ Multivariate } \\
\hline & & HR $(95 \% \mathrm{CI})$ & $p$ value & HR $(95 \%$ CI $)$ & $p$ value \\
\hline \multirow[t]{6}{*}{ Clinical data } & Body weight (kg) & & & & \\
\hline & $<80$ & 1.00 (ref) & & & \\
\hline & $\geq 80$ & $0.76(0.42-1.37)$ & 0.362 & & \\
\hline & Body Mass Index (BMI) & & & & \\
\hline & $<26.9$ & 1.00 (ref) & & & \\
\hline & $\geq 26.9$ & $1.41(0.78-2.53)$ & 0.244 & & \\
\hline \multirow[t]{15}{*}{ Cross-sectional data } & Skeletal muscle index $\left(\mathrm{SMI}, \mathrm{cm}^{2} / \mathrm{m}^{2}\right)$ & & & & \\
\hline & $<33.7$ & 1.00 (ref) & & & \\
\hline & $\geq 33.7$ & $0.64(0.35-1.16)$ & 0.147 & & \\
\hline & SM Area $\left(\mathrm{cm}^{2}\right)$ & & & & \\
\hline & $<102.8$ & 1.00 (ref) & & & \\
\hline & $\geq 102.8$ & $0.44(0.24-0.79)$ & 0.007 & & \\
\hline & Adipose tissue area & & & & \\
\hline & $<423.3$ & 1.00 (ref) & & & \\
\hline & $\geq 423.3$ & $0.82(0.46-1.47)$ & 0.521 & & \\
\hline & Visceral-to-subcutaneous fat ratio (VSR) & & & & \\
\hline & $<1.32$ & 1.00 (ref) & & & \\
\hline & $\geq 1.32$ & $1.40(0.78-2.51)$ & 0.258 & & \\
\hline & Visceral fat-to-muscle ratio (VMR) & & & & \\
\hline & $<2.24$ & 1.00 (ref) & & & \\
\hline & $\geq 2.24$ & $1.28(0.71-2.29)$ & 0.406 & & \\
\hline \multirow[t]{15}{*}{ 3D computational data } & Psoas Volume $\left(\mathrm{cm}^{3}\right)$ & & & & \\
\hline & $<165.4$ & 1.00 (ref) & & & \\
\hline & $\geq 165.4$ & $0.63(0.35-1.15)$ & 0.137 & & \\
\hline & Psoas HUmean & & & & \\
\hline & $<29.5$ & 1.00 (ref) & & 1.00 (ref) & \\
\hline & $\geq 29.5$ & $0.53(0.29-0.97)$ & 0.040 & $0.44(0.23-0.82)$ & 0.010 \\
\hline & Psoas HUmean Standard Deviation (DS) & & & & \\
\hline & $<39.6$ & 1.00 (ref) & & & \\
\hline & $\geq 39.6$ & $1.42(0.78-2.57)$ & 0.246 & & \\
\hline & Psoas SUVmax & & & & \\
\hline & $<3.9$ & 1.00 (ref) & & $1.00(\mathrm{ref})$ & \\
\hline & $\geq 3.9$ & $2.51(1.37-4.57)$ & 0.003 & $2.95(1.57-5.53)$ & 0.001 \\
\hline & Psoas SUVmean & & & & \\
\hline & $<1.1$ & 1.00 (ref) & & & \\
\hline & $\geq 1.1$ & $1.73(0.96-3.11)$ & 0.067 & & \\
\hline
\end{tabular}

Statistically significant differences were indicated in bold

assessing the sarcopenic status before treatment may guide customised strategies and support tailored treatment decision-making. A robust prognostic impact of sarcopenia has also been shown in $\mathrm{PC}$, in which this condition is present in over $60 \%$ of patients $[21,45,46]$. However, at the later stages of the disease (i.e., in heavily pre-treated mCRPC), the prevalence of sarcopenia can be considerably increased $[21,45]$. Coherently, in the present study, all the enrolled patients met the radiological criteria for sarcopenia. This finding suggests that the use of the conventional radiological definition of sarcopenia may be an inaccurate prognosticator at these stages. On these bases, we extended the conventional anthropometric evaluation of body composition to a vast range of cross-sectional and computational parameters.

From the methodological point of view, this approach allowed us to observe that the 3D computational analysis of SM overcomes the cross-sectional assessment in terms of the prediction of the long-term OS. This difference might be related to the higher reproducibility of volumetric methods compared to cross-sectional area measures, given to the 
Fig. 2 Kaplan-Meier curves for OS according to SM attenuation, metabolic metrics and their combination in the Attenuation Metabolic Index (AMI). Kaplan-Meier curves for overall survival (OS) according to SM HUmean (Panel A), SM SUVmax (Panel B), and their combination in the Attenuation Metabolic Index (AMI, Panel C)
A

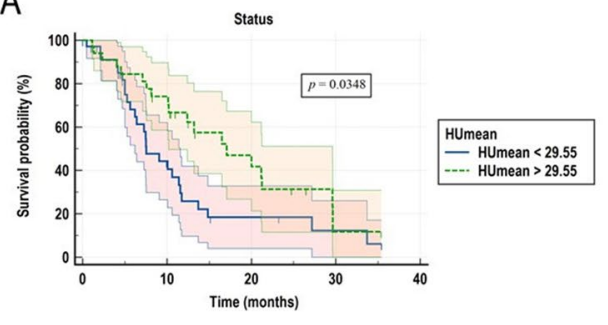

B

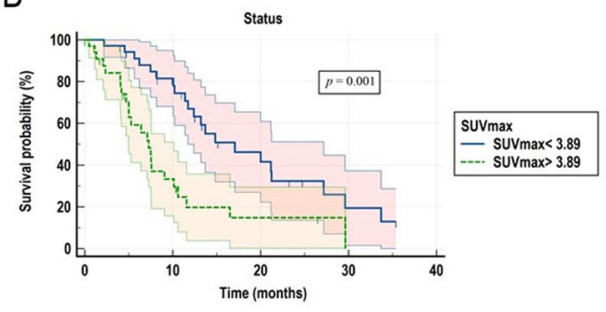

C

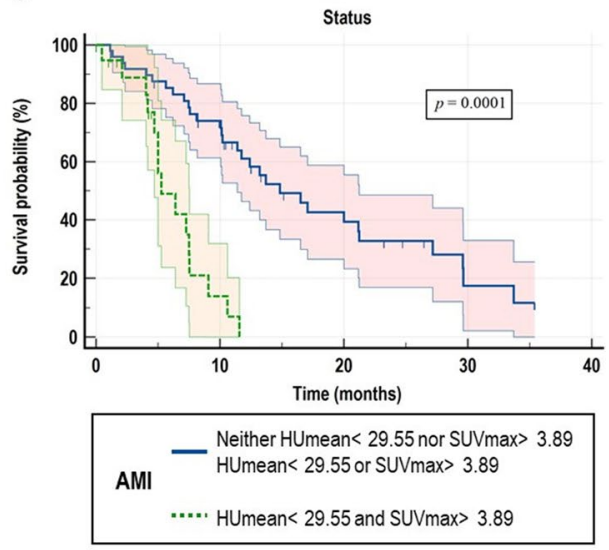

operator-independency of the latter approach. Further, imaging measures from a single axial section may not be representative of measures derived using volumetric analyses, as already documented in other solid tumours [47] as well as in anthropometric evaluations [48]. Finally, the computational analysis allowed us to extract FDG PET/CT-derived parameters from SM volumes.

The observed prognostic power of FDG-based measures of SM is of potential interest from the pathophysiological perspective. Indeed, it has been suggested that the reduction of SM HUmean in mCRPC (reflecting the increase in fat content) may result from the prolonged androgen deprivation, as castrate levels of testosterone may lead to reduced muscle mass and increased subcutaneous and visceral adipose tissue [49]. However, given the observed prognostic value of the increased FDG uptake in the sarcopenic SM, we presume that this phenomenon might also involve the occurrence of SM inflammation. Indeed, several previous studies highlighted the occurrence of a systemic inflamed state in heavily pretreated mCRPC $[11,17,39,50]$. On the other hand, a direct causal role of inflammation has been shown in the age-related decline of SM mass [51]. Consistently, a few previous studies correlated SM FDG uptake with clinical conditions possibly mediated through inflammatory mechanisms [52-55]. On the one side, the inflamed SM might be FDG-avid due to the high content in inflammatory infiltrates. Alternatively, given the oxidative environment promoted by SM inflammation [56], the increased FDG uptake may at least partially reflect the activation of NADPH generation by a pentose phosphate pathway selectively located within the endoplasmic reticulum, as previously documented in cancer cells $[57,58]$, neurons [59], astrocytes [60], cardiomyocytes [61], and, more importantly, in the SM $[62,63]$.
The present study has some limitations. First, as in any retrospective study we cannot a priori exclude the eventual occurrence of selection biases. However, we enrolled a naturalistic group of mCRPC patients' candidates to receive Radium-223 aiming to reflect the real-world clinical practice. This choice allowed us to identify a cohort of mCRPC with a remarkably high prevalence of sarcopenia. This result might be related to the late stage of the disease of patients receiving Radium-223 in the clinical practice, which is at least partially related to the restriction use promoted by the European Medical Agency (EMA) in 2018 [64]. However, we cannot assume the same prevalence of sarcopenia as generalizable to a broader group of mCRPC patients. Similarly, given the retrospective enrolment we are unable to estimate the exact duration of ADT prior to initiation of Radium-223 in enrolled patients. Owing to the documented correlation between ADT duration and SM loss [49] further studies are warranted to estimate the impact of this variable on obtained results. As a final consideration, CT-derived SM metrics were obtained from the low-dose CT of FDG PET/CT studies instead of using high-resolution CT. However, given the results of a recent study by Albano et al. [65], showing a strong correlation between SM metrics derived from lowand high-dose CT, we assume that this choice might have had a low impact on the reproducibility of our results.

\section{Conclusion}

The present data suggest that assessing individual patients SM metrics through an operator-independent computational analysis of FDG PET/CT data may potentially guide customised strategies and support tailored treatment decision-making in mCRPC patients candidates to receive Radium-223. 
Table 3 Uni- and multivariable Cox regression analyses including clinical, lab and imaging data

\begin{tabular}{|c|c|c|c|c|}
\hline \multirow[t]{2}{*}{ Variables } & \multicolumn{2}{|l|}{ Univariate } & \multicolumn{2}{|l|}{ Multivariate } \\
\hline & $\operatorname{HR}(95 \% \mathrm{CI})$ & $p$ value & $\mathrm{HR}(95 \% \mathrm{CI})$ & $p$ value \\
\hline \multicolumn{5}{|l|}{ PSA at diagnosis } \\
\hline$<12.34 \mathrm{ng} / \mathrm{mL}$ & 1.00 (ref) & & & \\
\hline$\geq 12.34 \mathrm{ng} / \mathrm{mL}$ & $0.72(0.39-1.31)$ & 0.289 & & \\
\hline \multicolumn{5}{|c|}{ International Society of Urological Pathology (ISUP) grade group } \\
\hline $\mathrm{GS}<8$ & 1.00 (ref) & & & \\
\hline $\mathrm{GS} \geq 8$ & $1.56(0.82-3.06)$ & 0.189 & & \\
\hline \multicolumn{5}{|c|}{ Metastases at diagnosis } \\
\hline No & 1.00 (ref) & & & \\
\hline Yes & $1.03(0.57-1.86)$ & 0.921 & & \\
\hline \multicolumn{5}{|c|}{ Previous chemotherapy } \\
\hline No & 1.00 (ref) & & & \\
\hline Yes & $0.96(0.53-1.74)$ & 0.906 & & \\
\hline \multicolumn{5}{|c|}{ Number of previous lines of therapy for CRPC } \\
\hline 1 & 1.00 (ref) & & & \\
\hline 2 & $0.91(0.01-3.53)$ & & & \\
\hline$\geq 3$ & $1.06(0.01-3.33)$ & 0.963 & & \\
\hline \multicolumn{5}{|c|}{ PSA at the time of FDG PET/CT } \\
\hline$<52.64 \mathrm{ng} / \mathrm{mL}$ & 1.00 (ref) & & 1.00 (ref) & \\
\hline$\geq 52.64 \mathrm{ng} / \mathrm{mL}$ & $3.48(1.79-6.73)$ & 0.0001 & $2.72(1.25-5.90)$ & 0.011 \\
\hline \multicolumn{5}{|c|}{ ALP at the time of FDG PET/CT } \\
\hline$<110 \mathrm{IU} / \mathrm{L}$ & 1.00 (ref) & & 1.00 (ref) & \\
\hline$\geq 110 \mathrm{IU} / \mathrm{L}$ & $5.61(2.50-12.54)$ & 0.0001 & $2.07(1.01-4.29)$ & 0.049 \\
\hline \multicolumn{5}{|c|}{$\mathrm{LDH}$ at the time of FDG PET/CT } \\
\hline$<229 \mathrm{IU} / \mathrm{L}$ & 1.00 (ref) & & & \\
\hline$\geq 229 \mathrm{IU} / \mathrm{L}$ & $2.46(1.26-4.783)$ & 0.008 & & \\
\hline \multicolumn{5}{|c|}{ Hemoglobin at the time of FDG PET/CT } \\
\hline$\geq 11.7 \mathrm{~g} / \mathrm{dL}$ & 1.00 (ref) & & & \\
\hline$<11.7 \mathrm{~g} / \mathrm{dL}$ & $3.12(1.64-5.89)$ & 0.001 & & \\
\hline \multicolumn{5}{|c|}{ NLR at the time of FDG PET/CT } \\
\hline$<3.73$ & 1.00 (ref) & & & \\
\hline$\geq 3.73$ & $1.39(0.76-2.54)$ & 0.285 & & \\
\hline \multicolumn{5}{|l|}{ MTV } \\
\hline$<250.8 \mathrm{~cm}^{3}$ & 1.00 (ref) & & & \\
\hline$\geq 250.8 \mathrm{~cm}^{3}$ & $2.51(1.37-4.61)$ & 0.003 & & \\
\hline \multicolumn{5}{|l|}{ TLG } \\
\hline$<771$ & 1.00 (ref) & & & \\
\hline$\geq 771$ & $2.56(1.37-4.76)$ & 0.003 & & \\
\hline \multicolumn{5}{|c|}{ Attenuation Metabolic Index (AMI) } \\
\hline Low risk & 1.00 (ref) & & 1.00 (ref) & \\
\hline High risk & $5.19(2.47-10.09)$ & 0.0001 & $3.12(1.30-7.47)$ & 0.010 \\
\hline
\end{tabular}

Statistically significant differences were indicated in bold
Author contributions Conceptualization: MB; data collection: $\mathrm{MB}$, RL, FD, AM, MID, CC, SR, SC, FL, CM, SER, EZ, GF, SM, CM; computational analysis: RL, CC, MP; statistical analysis: MB; writingoriginal draft preparation: $\mathrm{MB}$; writing-review and editing: all coauthors; funding acquisition: MB. All authors have read and agreed to the published version of the manuscript.
Funding MB was supported by grants from the Italian Ministry of Health $-5 \times 1000$ funds 2018-2019.

Availability of data and material The data that support the findings of this study are available from the corresponding author upon reasonable request. 


\section{Declarations}

Conflict of interest The authors do not have any conflict of interest to declare.

Ethical approval The local ethical committee approved the retrospective monocentric study.

Consent to participate All patients enrolled in the study signed a written informed consent at the time of FDG PET/CT and at the time of each Radium-223 administration, encompassing the use of anonymized data for retrospective research purposes.

Open Access This article is licensed under a Creative Commons Attribution 4.0 International License, which permits use, sharing, adaptation, distribution and reproduction in any medium or format, as long as you give appropriate credit to the original author(s) and the source, provide a link to the Creative Commons licence, and indicate if changes were made. The images or other third party material in this article are included in the article's Creative Commons licence, unless indicated otherwise in a credit line to the material. If material is not included in the article's Creative Commons licence and your intended use is not permitted by statutory regulation or exceeds the permitted use, you will need to obtain permission directly from the copyright holder. To view a copy of this licence, visit http://creativecommons.org/licenses/by/4.0/.

\section{References}

1. Siegel DA, O'Neil ME, Richards TB, Dowling NF, Weir HK. Prostate cancer incidence and survival, by stage and race/ethnicity - United States, 2001-2017. MMWR Morb Mortal Wkly Rep. 2020;69:1473-80.

2. Facchini G, Perri F, Misso G, D'Aniello C, Scarpati GDV, Rossetti $\mathrm{S}$, et al. Optimal management of prostate cancer based on its natural clinical history. Curr Cancer Drug Targets. 2018;18:457-67.

3. Morris MJ, Corey E, Guise TA, Gulley JL, Kevin Kelly W, Quinn DI, et al. Radium-223 mechanism of action: implications for use in treatment combinations. Nat Rev Urol. 2019;16:745-56.

4. Nelson BJB, Andersson JD, Wuest F. Targeted alpha therapy: progress in radionuclide production, radiochemistry, and applications. Pharmaceutics. 2020;13:49.

5. Ferrier MG, Radchenko V. An appendix of radionuclides used in targeted alpha therapy. J Med Imaging Radiat Sci. 2019;50:S58-65.

6. Cheetham PJ, Petrylak DP. Alpha particles as radiopharmaceuticals in the treatment of bone metastases: mechanism of action of radium-223 chloride (Alpharadin) and radiation protection. Oncology (Williston Park). 2012;26:330-7.

7. Flux GD. Imaging and dosimetry for radium-223: the potential for personalized treatment. Br J Radiol. 2017;90:20160748.

8. Pacilio M, Ventroni G, De Vincentis G, Cassano B, Pellegrini R, Di Castro E, et al. Dosimetry of bone metastases in targeted radionuclide therapy with alpha-emitting (223)Ra-dichloride. Eur J Nucl Med Mol Imaging. 2016;43:21-33.

9. Sciuto R, Rea S, Ungania S, Testa A, Dini V, Tabocchini MA, et al. The role of dosimetry and biological effects in metastatic castration-resistant prostate cancer (mCRPC) patients treated with 223Ra: first in human study. J Exp Clin Cancer Res. 2021;40:281.

10. Parker C, Nilsson S, Heinrich D, Helle SI, O'Sullivan JM, Fosså $\mathrm{SD}$, et al. Alpha emitter radium-223 and survival in metastatic prostate cancer. N Engl J Med. 2013;369:213-23.
11. Parikh S, Murray L, Kenning L, Bottomley D, Din O, Dixit $\mathrm{S}$, et al. Real-world outcomes and factors predicting survival and completion of Radium 223 in metastatic castrate-resistant prostate cancer. Clin Oncol (R Coll Radiol). 2018;30:548-55.

12. Wong WW, Anderson EM, Mohammadi H, Daniels TB, Schild SE, Keole SR, et al. Factors associated with survival following Radium-223 treatment for metastatic castration-resistant prostate cancer. Clin Genitourin Cancer. 2017;15:e969-75.

13. Frantellizzi V, Farcomeni A, Follacchio GA, Pacilio M, Pellegrini R, Pani R, et al. A 3-variable prognostic score (3-PS) for overall survival prediction in metastatic castration-resistant prostate cancer treated with 223Radium-dichloride. Ann Nucl Med. 2018;32:142-8.

14. Den RB, George D, Pieczonka C, McNamara M. Ra-223 Treatment for bone metastases in castrate-resistant prostate cancer: practical management issues for patient selection. Am J Clin Oncol. 2019;42:399-406.

15. Bauckneht M, Bertagna F, Donegani MI, Durmo R, Miceli A, De Biasi V, et al. The prognostic power of 18F-FDG PET/CT extends to estimating systemic treatment response duration in metastatic castration-resistant prostate cancer (mCRPC) patients. Prostate Cancer Prostatic Dis. 2021. https://doi.org/ 10.1038/s41391-021-00391-8.

16. Bauckneht M, Capitanio S, Donegani MI, Zanardi E, Miceli A, Murialdo R, et al. Role of baseline and post-therapy $18 \mathrm{~F}$ FDG PET in the prognostic stratification of metastatic castration-resistant prostate cancer (mCRPC) patients treated with Radium-223. Cancers (Basel). 2019;12:31.

17. Bauckneht M, Rebuzzi SE, Signori A, Donegani MI, Murianni V, Miceli A, et al. The prognostic role of baseline metabolic tumor burden and systemic inflammation biomarkers in metastatic castration-resistant prostate cancer patients treated with Radium-223: a proof of concept study. Cancers (Basel). 2020;12:3213.

18. Lee JS, Lee HS, Ha JS, Han KS, Rha KH, Hong SJ, et al. Subcutaneous fat distribution is a prognostic biomarker for men with castration resistant prostate cancer. J Urol. 2018;200:114-20.

19. Baracos VE, Arribas L. Sarcopenic obesity: hidden muscle wasting and its impact for survival and complications of cancer therapy. Ann Oncol. 2018;29:ii1-9.

20. Ohtaka A, Aoki H, Nagata M, Kanayama M, Shimizu F, Ide H, et al. Sarcopenia is a poor prognostic factor of castration-resistant prostate cancer treated with docetaxel therapy. Prostate Int. 2019;7:9-14.

21. Stangl-Kremser J, Suarez-Ibarrola R, Andrea D, Korn SM, Pones M, Kramer G, et al. Assessment of body composition in the advanced stage of castration-resistant prostate cancer: special focus on sarcopenia. Prostate Cancer Prostatic Dis. 2020;23:309-15.

22. Fielding RA, Vellas B, Evans WJ, Bhasin S, Morley JE, Newman $\mathrm{AB}$, et al. Sarcopenia: an undiagnosed condition in older adults. Current consensus definition: prevalence, etiology, and consequences. International working group on sarcopenia. J Am Med Dir Assoc. 2011;12:249-56.

23. Kazemi-Bajestani SM, Mazurak VC, Baracos V. Computed tomography-defined muscle and fat wasting are associated with cancer clinical outcomes. Semin Cell Dev Biol. 2016;54:2-10.

24. Deans C, Wigmore SJ. Systemic inflammation, cachexia and prognosis in patients with cancer. Curr Opin Clin Nutr Metab Care. 2005;8:265-9.

25. AIOM Guidelines on Prostate Cancer 2021. https://www.aiom.it. Accessed 14 Nov 2021.

26. Boellaard R, Delgado-Bolton R, Oyen WJ, Giammarile F, Tatsch $\mathrm{K}$, Eschner W, et al. FDG PET/CT: EANM procedure guidelines for tumour imaging: version 2.0. Eur J Nucl Med Mol Imaging. 2015;42:328-54. 
27. Poeppel TD, Handkiewicz-Junak D, Andreeff M, Becherer A, Bockisch A, Fricke E, et al. EANM guideline for radionuclide therapy with radium-223 of metastatic castration-resistant prostate cancer. Eur J Nucl Med Mol Imaging. 2018;45:824-45.

28. Mullie L, Afilalo J. CoreSlicer: a web toolkit for analytic morphomics. BMC Med Imaging. 2019;19:15.

29. Fearon K, Strasser F, Anker SD, Bosaeus I, Bruera E, Fainsinger RL, et al. Definition and classification of cancer cachexia: an international consensus. Lancet Oncol. 2011;12:489-95.

30. Beltrametti MC, Massone AM, Piana M. Hough transform of special classes of functions. SIAM J Imag Sci. 2013;6:391-412.

31. Bauckneht M, Lai R, Miceli A, Schenone D, Cossu V, Donegani MI, et al. Spinal cord hypermetabolism extends to skeletal muscle in amyotrophic lateral sclerosis: a computational approach to [18F]-fluorodeoxyglucose PET/CT images. EJNMMI Res. 2020;10:23.

32. Kruse V, Mees G, Maes A, D’Asseler Y, Borms M, Cocquyt V, et al. Reproducibility of FDG PET based metabolic tumor volume measurements and of their FDG distribution within. Q J Nucl Med Mol Imaging. 2015;59:462-8.

33. Kaplan EL, Meier P. Nonparametric estimation from incomplete observations. J Am Stat Assoc. 1958;53:457-81.

34. van der Zande K, Oyen WJG, Zwart W, Bergman AM. Radium-223 treatment of patients with metastatic castration resistant prostate cancer: biomarkers for stratification and response evaluation. Cancers (Basel). 2021;13:4346.

35. McKay RR, Jacobus S, Fiorillo M, Ledet EM, Cotogna PM, Steinberger AE, et al. Radium-223 use in clinical practice and variables associated with completion of therapy. Clin Genitourin Cancer. 2017;15:e289-98.

36. Saad F, Carles J, Gillessen S, Heidenreich A, Heinrich D, Gratt $\mathrm{J}$, et al. Radium-223 and concomitant therapies in patients with metastatic castration-resistant prostate cancer: an international, early access, open-label, single-arm phase $3 \mathrm{~b}$ trial. Lancet Oncol. 2016;17:1306-16.

37. Sartor O, Coleman RE, Nilsson S, Heinrich D, Helle SI, O'Sullivan JM, et al. An exploratory analysis of alkaline phosphatase, lactate dehydrogenase, and prostate-specific antigen dynamics in the phase 3 ALSYMPCA trial with radium-223. Ann Oncol. 2017;28:1090-7.

38. Prelaj A, Rebuzzi SE, Buzzacchino F, Pozzi C, Ferrara C, Frantellizzi V, et al. Radium-223 in patients with metastatic castrationresistant prostate cancer: efficacy and safety in clinical practice. Oncol Lett. 2019;17:1467-76.

39. Maruzzo M, Basso U, Borsatti E, Evangelista L, Alongi F, Caffo $\mathrm{O}$, et al. Results from a large, multicenter, retrospective analysis on Radium 223 use in metastatic castration-resistant prostate cancer (mCRPC) in the Triveneto Italian Region. Clin Genitourin Cancer. 2019;17:e187-94.

40. Parimi S, Tsang E, Alexander A, Mckenzie M, Bachand F, Sunderland $\mathrm{K}$, et al. A population-based study of the use of radium 223 in metastatic castration-resistant prostate cancer: factors associated with treatment completion. Can Urol Assoc J. 2017;11:350-5.

41. De Luca R, Costa RP, Tripoli V, Murabito A, Cicero G. The clinical efficacy of Radium-223 for bone metastasis in patients with castration-resistant prostate cancer: an Italian clinical experience. Oncology. 2018;94:161-6.

42. Shachar SS, Williams GR, Muss HB, Nishijima TF. Prognostic value of sarcopenia in adults with solid tumours: a meta-analysis and systematic review. Eur J Cancer. 2016;57:58-67.

43. Simonsen C, de Heer P, Bjerre ED, Suetta C, Hojman P, Pedersen BK, et al. Sarcopenia and postoperative complication risk in gastrointestinal surgical oncology: a meta-analysis. Ann Surg. 2018;268:58-69.

44. Bas O, Erdemir AG, Onur MR, Ozer N, Sener YZ, Aksu S, et al. Sarcopenia and anthracycline cardiotoxicity in patients with cancer. BMJ Support Palliat Care. 2021. https://doi.org/ 10.1136/bmjspcare-2021-003197.

45. Meyer HJ, Wienke A, Surov A. CT-defined low-skeletal muscle mass as a prognostic marker for survival in prostate cancer: a systematic review and meta-analysis. Urol Oncol. 2021;:S1078-1439(21):00368-9.

46. Jahrreiss V, Laukhtina E, D'Andrea D, Shariat SF. The prognostic value of sarcopenia in patients with prostate cancer: a systematic review. Curr Opin Urol. 2021;31:315-23.

47. Frenette A, Morrell J, Bjella K, Fogarty E, Beal J, Chaudhary V. Do Diametric measurements provide sufficient and reliable tumor assessment? An evaluation of diametric, areametric, and volumetric variability of lung lesion measurements on computerized tomography scans. J Oncol. 2015;2015:632943.

48. Foster B, Boutin RD, Lenchik L, Gedeon D, Liu Y, Nittur V, et al. Skeletal muscle metrics on clinical 18F-FDG PET/CT predict health outcomes in patients with sarcoma. J Nat Sci. 2018;4:e502.

49. Saylor PJ, Smith MR. Metabolic complications of androgen deprivation therapy for prostate cancer. J Urol. 2013;189:S34-42.

50. Bauckneht M, Rebuzzi SE, Signori A, Frantellizzi V, Murianni $\mathrm{V}$, Lodi Rizzini E, et al. The prognostic power of inflammatory indices and clinical factors in metastatic castration-resistant prostate cancer patients treated with radium-223 (BIO-Ra study). Eur J Nucl Med Mol Imaging. 2021. https://doi.org/10.1007/ s00259-021-05550-6.

51. Schaap LA, Pluijm SM, Deeg DJ, Harris TB, Kritchevsky SB, Newman AB, et al. Higher inflammatory marker levels in older persons: associations with 5-year change in muscle mass and muscle strength. J Gerontol A Biol Sci Med Sci. 2009;64:1183-9.

52. Kim JY, Jun DW, Choi J, Nam E, Son D, Choi YY. Psoas muscle fluorine-18-labelled fluoro-2-deoxy-d-glucose uptake associated with the incidence of existing and incipient metabolic derangement. J Cachexia Sarcopenia Muscle. 2019;10:894-902.

53. Zoico E, Corzato F, Bambace C, Rossi AP, Micciolo R, Cinti $\mathrm{S}$, et al. Myosteatosis and myofibrosis: relationship with aging, inflammation and insulin resistance. Arch Gerontol Geriatr. 2013;57:411-6.

54. Tateyama M, Fujihara K, Misu T, Arai A, Kaneta T, Aoki M. Clinical values of FDG PET in polymyositis and dermatomyositis syndromes: imaging of skeletal muscle inflammation. BMJ Open. 2015;5:e06763.

55. Tanaka S, Ikeda K, Uchiyama K, Iwamoto T, Sanayama Y, Okubo A, et al. [18F]FDG uptake in proximal muscles assessed by PET/CT reflects both global and local muscular inflammation and provides useful information in the management of patients with polymyositis/dermatomyositis. Rheumatology (Oxford). 2013;52:1271-8.

56. Foreman NA, Hesse AS, Ji LL. Redox signaling and sarcopenia: searching for the primary suspect. Int J Mol Sci. 2021;22:9045.

57. Marini C, Ravera S, Buschiazzo A, Bianchi G, Orengo AM, Bruno $S$, et al. Discovery of a novel glucose metabolism in cancer: the role of endoplasmic reticulum beyond glycolysis and pentose phosphate shunt. Sci Rep. 2016;6:25092.

58. Scussolini M, Bauckneht M, Cossu V, Bruno S, Orengo AM, Piccioli P, et al. G6Pase location in the endoplasmic reticulum: implications on compartmental analysis of FDG uptake in cancer cells. Sci Rep. 2019;9:2794.

59. Cossu V, Marini C, Piccioli P, Rocchi A, Bruno S, Orengo AM, et al. Obligatory role of endoplasmic reticulum in brain FDG uptake. Eur J Nucl Med Mol Imaging. 2019;46:1184-96.

60. Marini C, Cossu V, Kumar M, Milanese M, Cortese K, Bruno S, et al. The role of endoplasmic reticulum in the differential endurance against Redox stress in cortical and spinal astrocytes from the newborn SOD1G93A mouse model of amyotrophic lateral sclerosis. Antioxidants (Basel). 2021;10:1392. 
61. Bauckneht M, Pastorino F, Castellani P, Cossu V, Orengo AM, Piccioli P, et al. Increased myocardial 18F-FDG uptake as a marker of Doxorubicin-induced oxidative stress. J Nucl Cardiol. 2020;27:2183-94.

62. Bauckneht M, Cossu V, Castellani P, Piccioli P, Orengo AM, Emionite L, et al. FDG uptake tracks the oxidative damage in diabetic skeletal muscle: an experimental study. Mol Metab. 2020;31:98-108.

63. Marini C, Cossu V, Bonifacino T, Bauckneht M, Torazza C, Bruno $\mathrm{S}$, et al. Mechanisms underlying the predictive power of high skeletal muscle uptake of FDG in amyotrophic lateral sclerosis. EJNMMI Res. 2020;10:76.

64. EMA. EMA Restricts Use of Prostate Cancer Medicine XOFIGO. Available online: https://www.ema.europa.eu/en/news/ema-restr icts-use-prostate-cancer-medicine-xofigo. Accessed 14 Nov 2021.
65. Albano D, Camoni L, Rinaldi R, Tucci A, Zilioli VR, Muzi C, et al. Comparison between skeletal muscle and adipose tissue measurements with high-dose CT and low-dose attenuation correction CT of 18F-FDG PET/CT in elderly Hodgkin lymphoma patients: a two-centre validation. Br J Radiol. 2021;94:20200672.

Publisher's Note Springer Nature remains neutral with regard to jurisdictional claims in published maps and institutional affiliations. 